\title{
Determining the influence of structure on hybridization using oligonucleotide arrays
}

Because of a printing error, Figure 4 in "Determining the influence of structure on hybridization using oligonucleotide arrays", by Kalim U. Mir $^{\star}$ and Edwin M. Southern was printed incorrectly. The entire figure and caption are below:

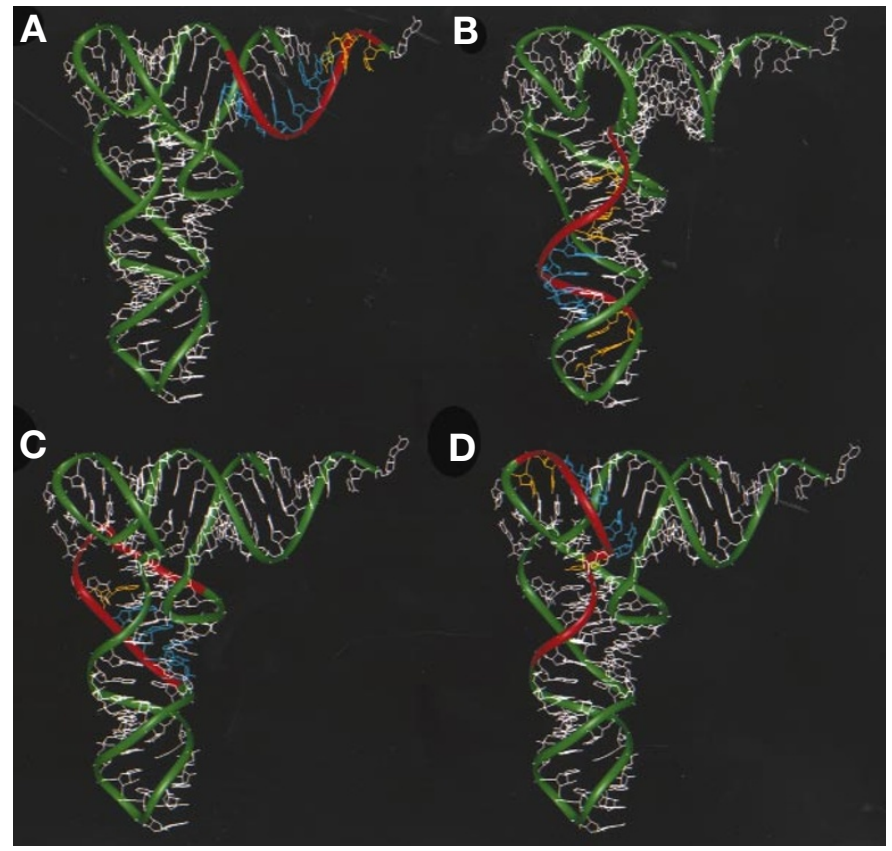

Figure 4. The tertiary structure of tRNA and helical regions that give high yield. A ribbon traces the RNA chain on wire-frame models of tRNA (derived from X-ray diffraction coordinates from the Protein Database). Positions of high yield in $3.5 \mathrm{M} \mathrm{TMACl}$ are marked red on the ribbon. Bases in single-stranded regions (yellow), which are stacked onto double-helical stem regions (blue), are marked on the wire-frame model. Bases in regions that give high yield have continuous helical order. (A) $3^{\prime}$ acceptor stem. From A66 to C72, bases are paired with the $5^{\prime}$ end of the RNA; from A73 to C75 they are unpaired but stacked onto the stem. (B) D stem/loop. There is continuous helical structure from A21 to C25; A21 is unpaired and stacks onto the stem. (C) Variable loop/anticodon stem. The helical system extends in both directions from the anticodon stem: A38 is stacked on the $5^{\prime}$ end and A44-G46 (A9 is intercalated between G46 and $\mathrm{mG} 45$ ) on the $3^{\prime}$ end. (D) TYC stem/variable loop. There is continuous helical structure from C48 to $\Psi 55$, which incorporates

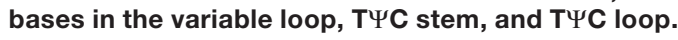

\section{Inducible isopentenyl transferase as a high-efficiency marker for plant transformation}

The corresponding author's e-mail address in "Inducible isopentenyl transferase as a high-efficiency marker for plant transformation" by Tim Kunkel, Qi-Wen Niu, Yang-Sun Chan, and Nam-Hai Chua was given incorrectly. The correct address is chua@rockvax.rockefeller.edu. 